\title{
The Online Prize-Collecting Facility Location Problem
}

\author{
Mário César San Felice ${ }^{\mathrm{a}, 1}$, Sin-Shuen Cheung b,2, \\ Orlando Lee ${ }^{\mathrm{a}, 3}$ and David P. Williamson ${ }^{\mathrm{b}, 4}$ \\ a Institute of Computing, Unicamp, Campinas SP, Brazil \\ b School of Operations Research and Information Engineering, Cornell University, \\ Ithaca $N Y$, USA
}

\begin{abstract}
In this paper we propose the Online Prize-Collecting Facility Location problem (OPCFL), which is an online version of the Prize-Collecting Facility Location problem (PCFL). The PCFL is a generalization of the Uncapacitated Facility Location problem (FL) in which some clients may be left unconnected by paying a penalty. Another way to think about it is that every client has a prize that can only be collected if it is connected. We give a primal-dual $\mathrm{O}(\log n)$-competitive algorithm for the OPCFL based on previous algorithms for Online Facility Location (OFL) due to Fotakis [3] and Nagarajan and Williamson [9].
\end{abstract}

Keywords: Online Algorithms, Competitive Analysis, Prize-Collecting Facility Location Problem, Primal-Dual Method.

\section{Introduction}

In the Facility Location problem (FL), we have a set of clients and a set of facilities in a metric space. Each facility has an associated opening cost. The

1 Partially supported by grant No. 2009/15535-1, São Paulo Research Foundation (FAPESP).

2 Partially supported by NSF grant CCF-1115256.

3 Partially supported by Bolsa de Produtividade do CNPq Proc. 303947/2008-0 and Edital Universal CNPq 477692/2012-5.

4 Partially supported by NSF grant CCF-1115256. 
cost of assigning a client to a facility is the distance between them. The goal of the problem is to select a set of facilities to open and to assign clients to open facilities so that the total cost of opening the facilities plus the cost of connecting clients to their assigned facilities is minimized. FL is an NP-complete problem that has been well-studied; several constant ratio approximation algorithms are known for it $[10,7,2,6]$. It is particularly interesting that several different design techniques, such as LP rounding, primal-dual and local search, are successful at achieving good approximation ratios for this problem.

The online version of FL is the Online Facility Location problem (OFL), in which the clients are revealed one at a time and each one needs to be connected to an open facility before the next one arrives. Throughout this process, no connection can be changed or opened facility can be closed. Algorithms for online problems are analyzed via competitive analysis [1]. An $\alpha$-competitive algorithm returns a solution whose cost is within a factor of $\alpha$ of the cost of an optimal solution to the corresponding offline problem; $\alpha$ is called the competitive ratio of the algorithm. There are randomized and deterministic $\mathrm{O}(\log n)$-competitive algorithms known for the OFL $[8,4,3,9,5]$, where $n$ is the number of clients. Moreover, the best lower bound for the competitive ratio of an algorithm for OFL is $\Omega\left(\frac{\log n}{\log \log n}\right)$ [4].

The Prize-Collecting Facility Location problem (PCFL) is a generalization of the FL in which some clients may be left unconnected by paying a penalty. Another way to think about it is that every client has a prize that can only be collected if it is connected. There is a constant ratio approximation algorithm known for $\mathrm{PCFL}$, due to $\mathrm{Xu}$ and $\mathrm{Xu}$ [11], that combines the primal-dual method with local search techniques.

Our Contributions. We propose the Online Prize-Collecting Facility Location problem (OPCFL), which is an online version of the PCFL. We give a primal-dual $\mathrm{O}(\log n)$-competitive algorithm for the OPCFL based on algorithms for OFL due to Fotakis [3] and Nagarajan and Williamson [9]. Since the OPCFL generalizes the OFL, the lower bound of $\Omega\left(\frac{\log n}{\log \log n}\right)$ applies to it.

\section{Preliminaries}

In this section we define the Online Prize-Collecting Facility Location problem (OPCFL) and present some notation and definitions that are useful later. Also, we show a linear programming relaxation for the OPCFL.

The OPCFL is a generalization of the OFL in which some clients may be left unconnected by paying a penalty. In general we use $i$ to denote a facility 
and $j$ to denote a client. When the arrival order matters we use $n(j)$ to denote the position of client $j$ in the sequence of clients. Also, when it is useful for the analysis we use indexed clients, i.e. $j_{k}$ to denote the $k$-th client that arrived. The dual variable $\alpha_{j}$, that is used in the algorithm, may be interpreted as the amount that client $j$ pays to open and connect to a facility, or to pay the penalty. When using indexed clients we use $\alpha_{k}$ for the dual variable of $j_{k}$.

The set of points in the metric space is $M$. The distance function is $d$, the penalty function for not connecting a client is $p$, and the opening cost function is $f$. If $j$ and $i$ are points in $M$, then $d(j, i)$ is the distance from $j$ to $i, p(j)$ is the penalty for leaving $j$ unconnected, and $f(i)$ is the cost for opening a facility at $i$. For $S \subseteq M$, let $d(j, S):=\min _{s \in S} d(j, s)$.

We denote the set of clients by $D$, the set of points in which a facility may be opened by $F$, and the set of open (or active) facilities by $F^{a}$. When the iteration of the algorithm matters, we use $D_{n(j)}$ and $F_{n(j)}^{a}$ to denote, respectively, the first $n(j)$ clients to arrive and the facilities opened by our algorithm to serve these clients. When using indexed clients we have $D_{k}=D_{n\left(j_{k}\right)}$ and $F_{k}^{a}=F_{n\left(j_{k}\right)}^{a}$. Thus, $F_{0}^{a}=D_{0}=\emptyset$. Also, we use $(v)^{+}:=\max \{0, v\}$, for any value $v$; and $a(j)$ to denote the facility to which client $j$ is connected by our algorithm. If $j$ is not connected, then $a(j)$ is null.

Next we present the linear programming relaxation for the OPCFL

$$
\begin{array}{ll}
\min & \sum_{i \in F} f(i) y_{i}+\sum_{j \in D} \sum_{i \in F} d(j, i) x_{j i}+\sum_{j \in D} p(j) z_{j} \\
\text { s.t. } & x_{j i} \leq y_{i} \quad \text { for } j \in D \text { and } i \in F, \\
& \sum_{i \in F} x_{j i}+z_{j} \geq 1 \quad \text { for } j \in D, \\
& y_{i} \geq 0, x_{j i} \geq 0, z_{j} \geq 0 \text { for } j \in D \text { and } i \in F,
\end{array}
$$

and its dual

$$
\begin{aligned}
& \max \sum_{j \in D} \alpha_{j} \\
& \text { s.t. } \sum_{j \in D}\left(\alpha_{j}-d(j, i)\right)^{+} \leq f(i) \text { for } i \in F \text {, } \\
& \alpha_{j} \leq p(j) \quad \text { for } j \in D \text {, } \\
& \alpha_{j} \geq 0 \quad \text { for } j \in D \text {. }
\end{aligned}
$$




\section{The Online Primal-Dual Algorithm}

In this section we describe a primal-dual algorithm for the OPCFL, that is based on Fotakis [3] and Nagarajan and Williamson [9] algorithms for OFL.

Input: $G=(V, E), d, f, p, F$

$D \leftarrow \emptyset ; F^{a} \leftarrow \emptyset ;$

while a new client $j^{\prime}$ arrives

increase $\alpha_{j^{\prime}}$ until one of the following happens:

(a) $\alpha_{j^{\prime}}=d\left(j^{\prime}, i\right)$ for some $i \in F^{a}$;

(b) $f(i)=\left(\alpha_{j^{\prime}}-d\left(j^{\prime}, i\right)\right)+\sum_{j \in D}\left(\min \left\{d\left(j, F^{a}\right), p(j)\right\}-d(j, i)\right)^{+}$ for some $i \in F \backslash F^{a}$;

(c) $\alpha_{j^{\prime}}=p\left(j^{\prime}\right)$ (in this case $i$ is chosen to be null, i.e. $\{i\}=\emptyset$ );

$F^{a} \leftarrow F^{a} \cup\{i\} ; D \leftarrow D \cup\left\{j^{\prime}\right\} ; a\left(j^{\prime}\right) \leftarrow i ;$

return $\left(F^{a}, a\right)$;

Our algorithm receives a graph $G=(V, E)$, with edge costs $d$, facility opening costs $f$, penalty costs $p$, and a set of nodes $F$ in which a facility may be opened.

Every time a new client $j^{\prime}$ arrives, we increase its dual variable $\alpha_{j^{\prime}}$ until: (a) $j^{\prime}$ is connected to an already opened facility, (b) a facility is opened and $j^{\prime}$ is connected to it, or (c) $j^{\prime}$ is not connected and pays a penalty $p\left(j^{\prime}\right)$.

The solution built by the algorithm consists of a set of open (or active) facilities $F^{a}$, and a function $a$ that assigns each client to an opened facility. If a client $j$ is not connected, then $a(j)$ is set to null.

\subsection{Algorithm Analysis}

In this subsection we analyze the competitivity of our algorithm.

Let $n=|D|, H_{n}$ be the $n$-th harmonic number, $D_{n}^{c}$ be the set of clients that the algorithm connected to some facility, and $D_{n}^{p}$ be the set of clients for which the algorithm paid the penalty. Notice that $D_{n}^{c} \cap D_{n}^{p}=\emptyset$. So, we have that $c(\mathrm{ALG}):=\sum_{i \in F_{n}^{a}} f(i)+\sum_{j \in D_{n}^{c}} d(j, a(j))+\sum_{j \in D_{n}^{p}} p(j)$ is the cost of the solution $\left(F^{a}, a\right)$ of our algorithm. Also, let $c(\mathrm{OPT})$ be the cost of the offline optimal solution.

Theorem $3.1 c(\mathrm{ALG}) \leq 6 H_{n} c(\mathrm{OPT})$.

This theorem relies on several other results. The main ones are described in what follows. Due to space constraints, the auxiliary results and the proofs are omitted.

First we bound the cost of the algorithm using the dual variables. 
Lemma $3.2 c(\mathrm{ALG}) \leq 2 \cdot \sum_{j \in D_{n}} \alpha_{j}$.

Now we bound the cost of the dual variables of the connected clients.

Lemma 3.3 Let $D^{c} \subseteq D_{n}^{c}$ be any subset of connected clients, and $i \in F$ be any place in which a facility may be opened. We have that:

$$
\frac{f(i)}{2} \geq \sum_{j \in D^{c}}\left(\frac{\alpha_{j}}{2 H_{\left|D_{n}\right|}}-d(j, i)\right) .
$$

Let $\alpha$ be the vector of the dual variables of a solution produced by our algorithm. For a dual solution to be feasible, its variables must respect all the dual constraints. The dual formulation of OPCFL has two sets of constraints. One is $\alpha_{j} \leq p(j)$ for any $j$ in $D$. Due to case (c) of the algorithm, $\alpha$ respects these constraints.

The other set of constraints is $\sum_{j \in D}\left(\alpha_{j}-d(j, i)\right)^{+} \leq f(i)$, for any $i$ in $F$. Let $\alpha^{c}$ be the vector of the dual variables corresponding to clients that are in $D_{n}^{c}$. Lemma 3.3 shows that if we divide $\alpha^{c}$ by $2 H_{\left|D_{n}\right|}$, the rescaled variables respect the dual constraints. Thus, we know how to make the dual variables $\alpha_{j}$ feasible for each client $j$ in $D_{n}^{c}$. It remains to show the same for the clients in $D_{n}^{p}$. That is what we do in the following theorem.

Theorem 3.4 Throughout the execution of the algorithm, the following inequality holds:

$$
\frac{f(i)}{3} \geq \sum_{j \in D^{p}}\left(\frac{\alpha_{j}}{3 H_{\left|D_{n}\right|}}-d(j, i)\right),
$$

for any $i \in F$ potential facility to open, and any subset of penalty clients $D^{p} \subseteq D_{n}^{p}$.

Let $\alpha^{p}$ be the vector of the dual variables corresponding to clients in $D_{n}^{p}$. Theorem 3.4 shows that if we divide $\alpha^{p}$ by $3 H_{\left|D_{n}\right|}$, the rescaled variables respect the dual constraints. Thus, we know how to make the dual variables $\alpha_{j}$ feasible for each client $j$ in $D_{n}^{p}$.

Proof. (Theorem 3.1). Using Lemmas 3.2 and 3.3, and Theorem 3.4, with the fact that, the cost of a feasible dual solution is a lower bound for the cost of any primal solution, we have:

$$
\begin{aligned}
c(\mathrm{ALG}) & \leq 2 \sum_{j \in D_{n}} \alpha_{j} \\
& =2 \sum_{j \in D_{n}^{c}} \alpha_{j}+2 \sum_{j \in D_{n}^{p}} \alpha_{j}
\end{aligned}
$$




$$
\begin{aligned}
& =4 H_{n} \sum_{j \in D_{n}^{c}} \frac{\alpha_{j}}{2 H_{n}}+6 H_{n} \sum_{j \in D_{n}^{p}} \frac{\alpha_{j}}{3 H_{n}} \\
& \leq 6 H_{n}\left(\sum_{j \in D_{n}^{c}} \frac{\alpha_{j}}{2 H_{n}}+\sum_{j \in D_{n}^{p}} \frac{\alpha_{j}}{3 H_{n}}\right) \leq 6 H_{n} c(\mathrm{OPT}) .
\end{aligned}
$$

\section{References}

[1] Borodin, A. and R. El-Yaniv, "Online Computation and Competitive Analysis," Press Syndicate of the University of Cambridge, 1998.

[2] Byrka, J. and K. Aardal, An optimal bifactor approximation algorithm for the metric facility location problem, SIAM Journal on Computing 39 (2010), pp. 2212-2231.

[3] Fotakis, D., A primal-dual algorithm for online non-uniform facility location, Journal of Discrete Algorithms 5 (2007), pp. 141-148.

[4] Fotakis, D., On the competitive ratio for online facility location, Algorithmica 50 (2008), pp. 1-57.

[5] Fotakis, D., Online and incremental algorithms for facility location, SIGACT News 42 (2011), pp. 97-131.

[6] Li, S., A 1.488 approximation algorithm for the uncapacitated facility location problem, Information and Computation 222 (2013), pp. 45-58.

[7] Mahdian, M., Y. Ye and J. Zhang, Approximation algorithms for metric facility location problems, SIAM Journal on Computing 36 (2006), pp. 411-432.

[8] Meyerson, A., Online facility location, in: Foundations of Computer Science, 42nd IEEE Symposium, FOCS 2001, 2001, pp. 426-431.

[9] Nagarajan, C. and D. P. Williamson, Offline and online facility leasing, Discrete Optimization 10 (2013), pp. 361-370.

[10] Shmoys, D. B., Approximation algorithms for facility location problems, in: Approximation Algorithms for Combinatorial Optimization, 3rd International Workshop, APPROX 2000, number 1913 in Lecture Notes in Computer Science (2000), pp. 27-33.

[11] Xu, G. and J. Xu, An improved approximation algorithm for uncapacitated facility location problem with penalties, Journal of Combinatorial Optimization 17 (2009), pp. 424-436. 